\title{
The Heisenberg antiferromagnet on the square-kagomé lattice
}

\author{
J. Richter ${ }^{1}$, J. Schulenburg ${ }^{2}$, P. Tomczak ${ }^{3}$, D. Schmalfuß ${ }^{1}$ \\ ${ }^{1}$ Institut für Theoretische Physik, Universität Magdeburg, P.O. Box 4120, D-39016 Magdeburg, Germany \\ 2 Universitätsrechenzentrum, Universität Magdeburg, P.O. Box 4120, D-39016 Magdeburg, Germany \\ 3 Physics Department, Adam Mickiewicz University, Umultowska 85, 61-614 Poznań, Poland
}

Received June 8, 2009

\begin{abstract}
We discuss the ground state, the low-lying excitations as well as high-field thermodynamics of the Heisenberg antiferromagnet on the two-dimensional square-kagomé lattice. This magnetic system belongs to the class of highly frustrated spin systems with an infinite non-trivial degeneracy of the classical ground state as it is also known for the Heisenberg antiferromagnet on the kagomé and on the star lattice. The quantum ground state of the spin-half system is a quantum paramagnet with a finite spin gap and with a large number of non-magnetic excitations within this gap. We also discuss the magnetization versus field curve that shows a plateaux as well as a macroscopic magnetization jump to saturation due to independent localized magnon states. These localized states are highly degenerate and lead to interesting features in the low-temperature thermodynamics at high magnetic fields such as an additional low-temperature peak in the specific heat and an enhanced magnetocaloric effect.
\end{abstract}

Key words: frustration, quantum Heisenberg antiferromagnet, quantum disorder, strong magnetic field

PACS: $75.10 . J m, 75.45 .+j, 75.60 . E j, 75.50 . E e$

\section{Introduction}

The magnetic properties of low-dimensional antiferromagnetic quantum spin systems have been a subject of many theoretical studies in recent years $[1,2]$. These studies are motivated by the recent progress in synthesizing quasi-two-dimensional magnetic materials which exhibit exciting quantum effects [3-10]. Even spin systems on more exotic frustrated lattices such as the star lattice [11,12], the maple-leaf lattice [13,11] and the triangulated kagomé lattice [14] have been synthesized recently [15-17].

A lot of activities in this area were focused on frustrated spin-half Heisenberg antiferromagnets like the $J_{1}-J_{2}$ antiferromagnet on the square lattice (see, e. g. [18-28] and references therein) and on three-dimensional cubic lattices [29-33], the Heisenberg antiferromagnet (HAFM) on the star lattice $[12,11,34]$ and last but not least the HAFM on the kagomé lattice (see the reviews $[11,35-$ 37] and references therein). Due to the extreme frustration, the HAFM on the kagomé and on the star lattices shows an infinite non-trivial degeneracy of the classical ground state. Furthermore, both spin lattices exhibit a magnetization jump to saturation due to localized magnon states $[11,12,38,39]$. Although there is most likely no magnetic ground state order for the quantum spinhalf HAFM on both lattices, the nature of both quantum ground states as well as the low-lying spectrum are basically different. It was argued [12] that the origin for this difference lies in the existence of nonequivalent nearest-neighbor (NN) bonds in the star lattice whereas all NN bonds in the kagomé lattice are equivalent. Another striking difference that is relevant to magnetic properties [40] lies in the number of spins in the unit cell which is odd for the kagomé lattice but even for the star lattice. As a result of the interplay between quantum fluctuations and strong frustration for the kagomé lattice, the ground state is most likely magnetically disordered with a (small) spin gap to the triplet excitations and an exceptional density of low-lying singlets below 
the first magnetic excitation. Various possible ground state phases, such as valence bond solids, gapped spin liquids of different kinds as well as stable critical phases are under discussion, see e. g. $[11,35,37,41-48]$. On the other hand, for the star lattice one meets a so-called explicit valence-bond crystal with a well-pronounced gap to all excitations which can be attributed to the nonequivalence of the NN bonds and to the even number of $s=1 / 2$ spins in the unit cell $[11,12,34]$.

In this paper we consider the spin-half HAFM on the square-kagomé [49-51] lattice (see figure 1). The square-kagomé lattice is built both by regular and non-regular polygons and it has two nonequivalent sites. Therefore, it does not belong to the class of the so-called uniform Archimedean tilings [11,52] (like, e. g. square, triangular, star or kagomé lattices). Nevertheless, there exist some important geometrical similarities both to the kagomé and to the star lattices. Similar to the kagomé lattice it has coordination number $z=4$, the even regular polygons (hexagons for the kagomé, squares for the square-kagomé lattice) are surrounded only by odd regular polygons (triangles) and both lattices contain corner sharing triangles. As a result, the HAFM on the square-kagomé lattice is also strongly frustrated and exhibits an infinite non-trivial degeneracy of the classical ground state, see section 3. The similarity to the star lattice consists in the existence of nonequivalent $\mathrm{NN}$ bonds and in the fact that both lattices have an even number of spins in the unit cell. Moreover, the classical ground state of the HAFM on the star lattice also exhibits an infinite non-trivial degeneracy. Due to these similarities we can expect that the HAFM on the square-kagomé lattice is another candidate for a quantum paramagnetic ground state. However, the question arises, whether the quantum ground state displays similar properties as that for the kagomé lattice or as that for the star lattice or for none of them.
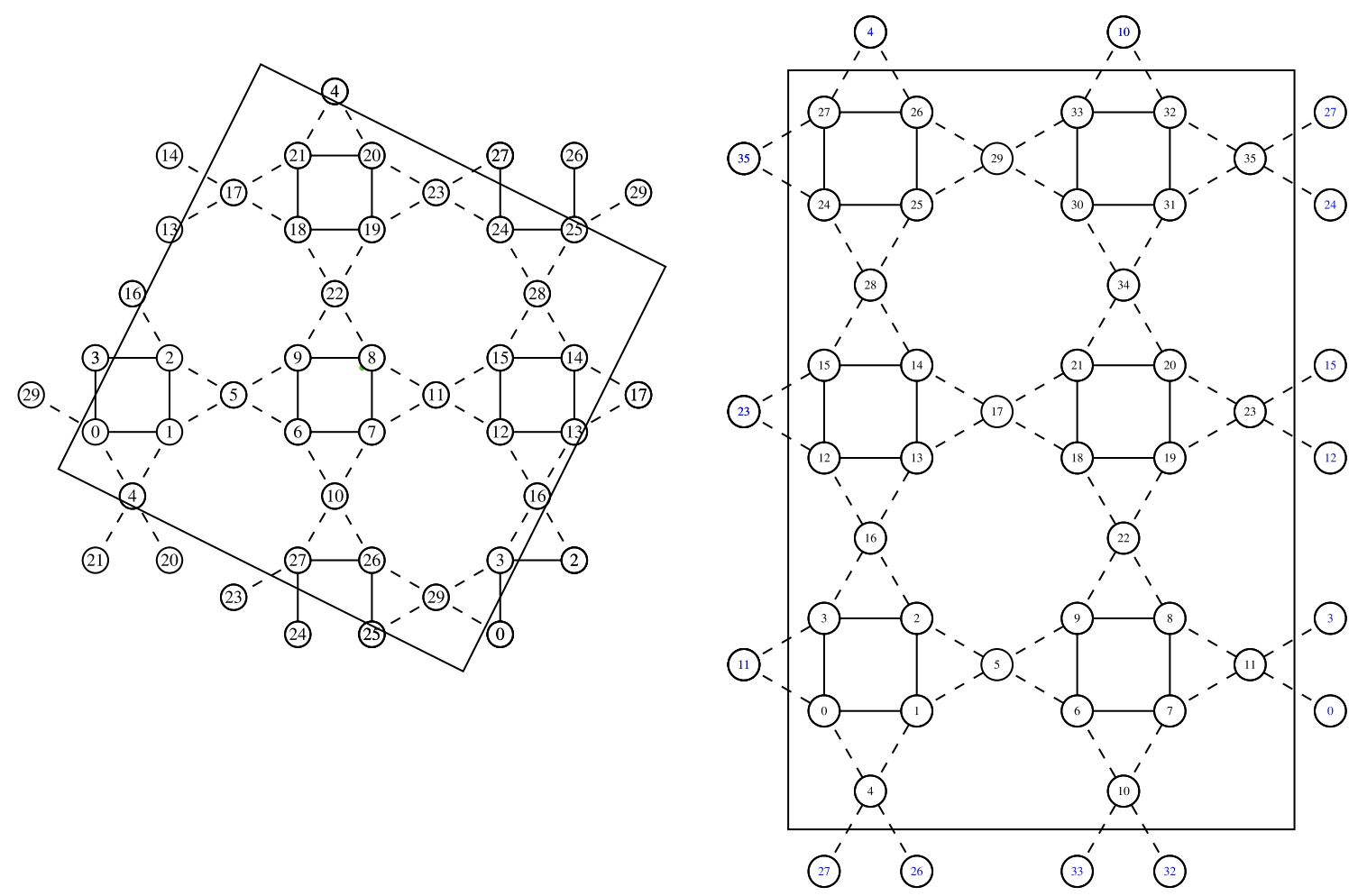

Figure 1. Two finite square-kagomé lattices with $N=30$ (left) and $N=36$ (right) sites. The two topologically nonequivalent nearest-neighbor bonds are distinguished by solid (square bonds $J_{\mathrm{S}}$ ) and dashed lines (triangular bonds $J_{\mathrm{T}}$ ). 


\section{The model}

The geometric unit cell of the square-kagomé lattice contains six sites and the underlying Bravais lattice is a square one (see figure 1). For this lattice we consider the spin-half HAFM in a magnetic field $h$

$$
\hat{H}=J \sum_{\langle i j\rangle} \mathbf{S}_{i} \cdot \mathbf{S}_{j}-h \hat{S}^{z}
$$

where the sum runs over pairs of neighboring sites $\langle i j\rangle$ and $\hat{S}^{z}=\sum_{i} \hat{S}_{i}^{z}$. As mentioned above the square-kagomé lattice carries topologically nonequivalent $\mathrm{NN}$ bonds $J_{\mathrm{S}}$ (square bonds, solid lines in figure 1) and $J_{\mathrm{T}}$ (triangular bonds, dashed lines in figure 1, see also figure 2). For the uniform lattice these bonds are of equal strength $J_{\mathrm{S}}=J_{\mathrm{T}}=J$ and we set $J=1$ in what follows.
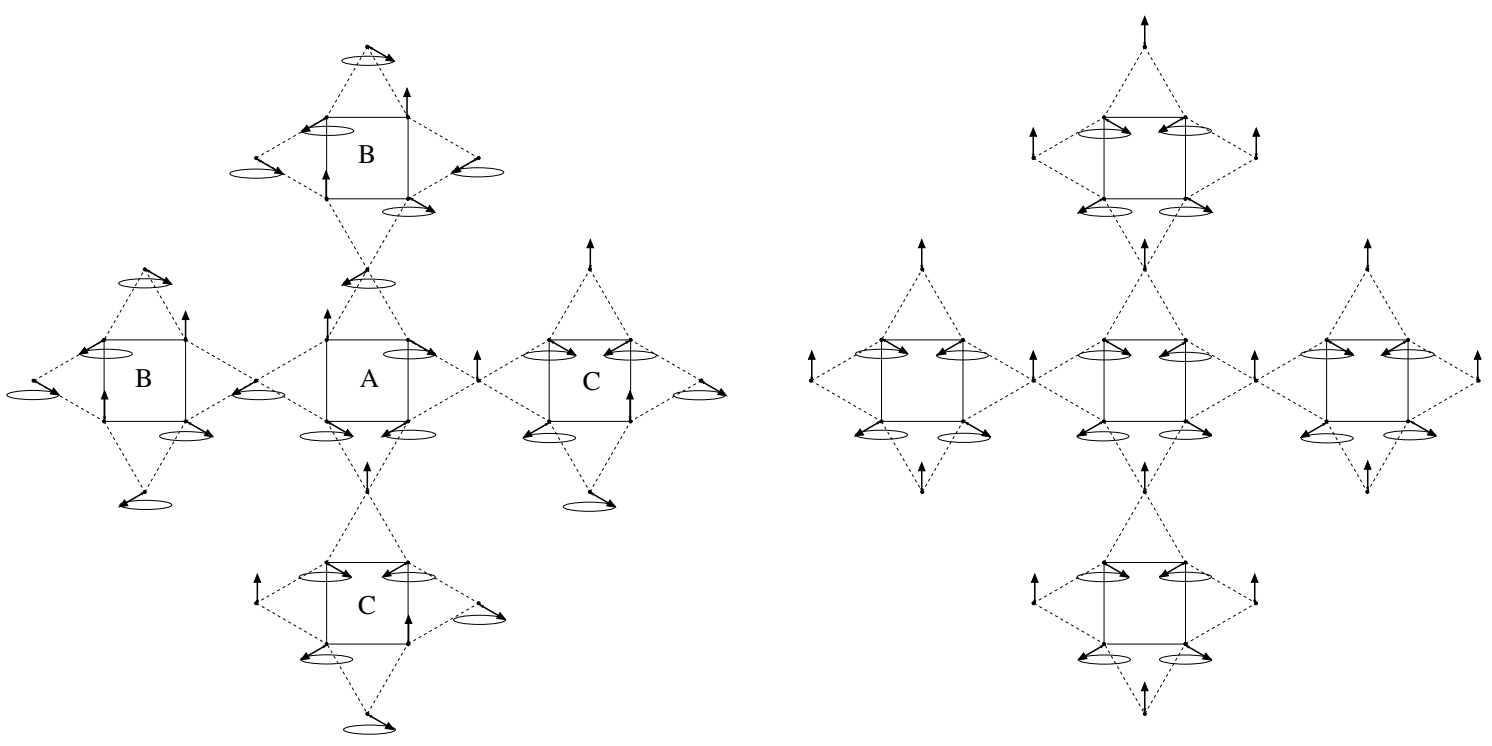

Figure 2. Two variants of the ground state of the classical HAFM on the square-kagomé lattice: The state on the left side has a magnetic unit cell which is three times as large as the geometric one and resembles the $\sqrt{3} \times \sqrt{3}$ state of the kagomé and the star lattices. For the state on the right side the magnetic unit cell is identical to the geometric one and corresponds to the $q=0$ state of the kagomé and the star lattices. The dotted ellipses show further degrees of freedom of the highly degenerate classical ground state.

\section{Semi-classical ground state}

In the classical ground state for $h=0$, the angle between neighboring spins is $2 \pi / 3$. Since the triangles are "corner sharing", there is a non-trivial infinite degeneracy resulting from the possible rotation of two spins on a triangle (see also figure 2). The classical ground state energy per bond is $e_{0}^{\text {class }}=-0.125$ assuming classical spin vectors of length $s=1 / 2$. Similar to the kagomé and the star lattices there are two variants of the classical ground state, shown in figure 2, being candidates for possible magnetic ground state ordering.

To discuss the effect of quantum fluctuations on a semiclassical level we perform a linear spinwave theory (LSWT) starting with the coplanar classical ground states. We have to consider six types of magnons according to the six sites per unit cell. As for the kagomé [53-55] and the star lattice [12] the spin-wave spectra are equivalent for all coplanar configurations satisfying the classical ground state constraint. We obtain six spin-wave branches, three optical branches, one acoustical and two dispersionless zero modes. Thus, flat zero modes appear as it is observed 
for the kagomé and star lattice case. Within the LSWT approximation there is no "order-bydisorder" selection among the coplanar classical ground states due to the equivalence of the spinwave branches, exactly like for the kagomé lattice [54,36] and the star lattice [12,34].

The ground state energy per bond for $s=1 / 2$ in the LSWT is $e_{0}=-0.236555$. Due to the flat zero modes, the integral for the sublattice magnetization diverges which might be understood as some hint for the absence of the classical order, cf. also [55] for the kagomé lattice. Although on the semiclassical LSWT level, the square-kagomé, the kagomé and the star lattices exhibit almost identical properties, the situation will change taking into account the quantum fluctuations more properly.

\section{The quantum ground state and low-lying excitations at zero field}

To take into account the quantum fluctuations going beyond the semiclassical LSWT we use Lanczos exact diagonalization (ED) to calculate the ground state and the lowest excitations for the $s=1 / 2$ HAFM at $h=0$ on finite lattices of $N=12,18,24,30$ and 36 sites with periodic boundary conditions. The finite lattices with $N=30$ and 36 are shown in figure 1 . The ground states of all those systems are singlets and the ground state energy per bond $e_{0}$ and the degeneracy of the quantum ground state $d_{\mathrm{GS}}$ are given in table 1 . Furthermore, we give in table 1 the gap to the first triplet excitation (spin gap) $\Delta$. Note that $e_{0}$ and $\Delta$ are significantly smaller than the corresponding values for the star lattice but of comparable size as the values for the kagomé lattice.

Table 1. Ground state energy per bond $e_{0}$, ground state degeneracy $d_{\mathrm{GS}}$, spin gap $\Delta$ and square of the order parameter $\left(\mathrm{m}^{+}\right)^{2}$ of the spin-half HAFM on finite square-kagomé lattices.

\begin{tabular}{lccccc}
\hline $\mathrm{N}$ & 12 & 18 & 24 & 30 & 36 \\
\hline$e_{0}\left(d_{\mathrm{GS}}\right)$ & $-0.226870(1)$ & $-0.223767(2)$ & $-0.224165(1)$ & $-0.221527(4)$ & $-0.222197(3)$ \\
$\Delta$ & 0.382668 & 0.290191 & 0.263906 & 0.188865 & 0.139550 \\
$\left(m^{+}\right)^{2}$ & 0.184160 & 0.116455 & 0.086735 & 0.068618 & 0.060475 \\
\hline
\end{tabular}

Now we compare the spin-spin correlations with those for the HAFM on the triangular, kagomé and star lattices in figure 3. For the triangular, kagomé and star lattices we consider the strongest correlations as a measure for magnetic order for the largest finite lattices accessible for ED and present in figure 2 the maximal absolute correlations $\left|\left\langle\mathbf{S}_{i} \mathbf{S}_{j}\right\rangle\right|_{\max }$ for a certain separation $R=\mid \mathbf{R}_{i}-$ $\mathbf{R}_{j} \mid$ versus $R$. Contrary to those lattices the square-kagomé lattice contains two nonequivalent sites. Hence, we present for the square-kagomé lattice all different correlations $\left|\left\langle\mathbf{S}_{i} \mathbf{S}_{j}\right\rangle\right|$ in figure 3 . Note further that we prefer to present the correlations for the finite square-kagomé lattice with $N=30$ sites, since it has better geometrical properties than the largest square-kagomé lattice considered $(N=36)$. As expected we have very rapidly decaying correlations for the disordered kagomé and star case, whereas the correlations for the Néel ordered triangular lattice are much stronger for larger distances and show a kind of saturation for larger $R$. The decay of the correlations for the square-kagomé lattice is also very rapid thus indicating the lack of long-range order in the spinspin correlation function. The two nonequivalent NN bonds carry very similar spin correlations, its difference for $N=30$ is only about $10 \%$, which is contrary to the star lattice where the two nonequivalent NN bonds differ by a factor of 3.5 [12].

Let us now discuss the low-lying spectrum of the square-kagomé lattice (see figure 4), following the lines of the discussion of the spectrum for the triangular [57], the kagomé lattice [41,42] and the star lattice $[12,34]$. The lowest states $E_{\min }(S)$ shown in figure 4 are not well described by the effective low-energy Hamiltonian $H_{\text {eff }} \sim E_{0}+\mathbf{S}^{2} / 2 N \chi_{0}$ of a semiclassically ordered system: One can see rather clearly that the dependence $E_{\min }(S)$ vs. $S(S+1)$ is not a linear one and there are no separated so-called quasi degenerate joint states [57] which in the thermodynamic limit could collapse to a ground state breaking the rotational symmetry. Note further that the symmetries of the lowest states in each sector of $S$ cannot be attributed to the classical ordered ground states 


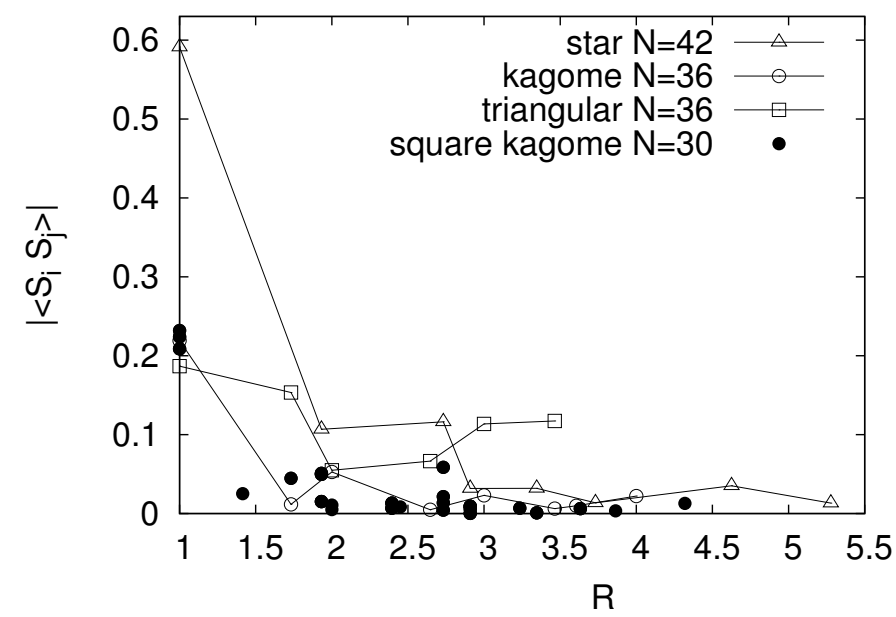

Figure 3. The absolute value of the spin-spin correlations $\left|\left\langle\mathbf{S}_{i} \mathbf{S}_{j}\right\rangle\right|$ versus $R=\left|\mathbf{R}_{i}-\mathbf{R}_{j}\right|$ for the HAFM on the square-kagomé $(N=30)$, the kagomé $(N=36)$, the star $(N=42)$ and the triangular $(N=36)$ lattices. For the kagomé, star and triangular lattices we present only the maximal values of $\left|\left\langle\mathbf{S}_{i} \mathbf{S}_{j}\right\rangle\right|$ for a certain separation $R$ (the lines are guides for the eyes), for the square-kagomé lattice we present all different values for $\left|\left\langle\mathbf{S}_{i} \mathbf{S}_{j}\right\rangle\right|$ obtained by averaging over the four degenerate ground states. Note that the data on for the kagomé lattice coincide with those of [56] and the data for the triangular and the star lattice with those of [12].

shown in figure 2. These features are similar to the kagomé [41,42] and the star [12] lattices. But there is one striking difference between the kagomé lattice and the star lattice. While the former one has an exponentially increasing number of non-magnetic singlets filling the singlet-triplet gap (spin gap) no such low-lying singlets were found for the star lattice $[12,41,42]$. This difference was attributed to the nonequivalence of NN bonds in the star lattice and the resulting dimerization of the ground state. Though the square-kagomé lattice has also nonequivalent NN bonds its spectrum is different from that of the star lattice, rather it shows, similar to the kagomé lattice, a large number $N_{\mathrm{s}}$ of non-magnetic excitations within the singlet-triplet gap. We find $N_{\mathrm{s}}=6(N=12)$, $13(N=18), 17(N=24), 47(N=30), 38(N=36)$. These numbers increase with growing size (except for $N=36$, which might be attributed to the lower symmetry of this finite lattice) but are smaller than those for the kagomé lattice [42], where an exponential increase of $N_{\mathrm{s}}$ with $N$ was suggested. Our data for the square-kagomé lattice do not provide a secure conclusion about a possible exponential increasing of $N_{\mathrm{s}}$ with $N$.

For the discussion of magnetic long-range order we use the following finite-system order parameter $[11,12]$

$$
m^{+}=\left(\frac{1}{N^{2}} \sum_{i, j}\left|\left\langle\mathbf{S}_{i} \mathbf{S}_{j}\right\rangle\right|\right)^{\frac{1}{2}},
$$

which is independent on any assumption of eventual classical order. The value $m_{\text {class }}^{+}$for the two ordered classical ground states shown in figure 2 is $m_{\text {class }}^{+}=\frac{1}{2} \sqrt{2 / 3}$, which is the same as for the classical $\sqrt{3} \times \sqrt{3}$ and $q=0$ states on the kagomé and on the star lattices.

The numerical values for $\left(m^{+}\right)^{2}$ are collected in table 1 . The values of $\left(m^{+}\right)^{2}$ for the squarekagomé lattice are comparable to those for the kagomé lattice but are slightly smaller than the corresponding values for the star lattice [12].

To estimate the values of $e_{0}, \Delta$ and $m^{+}$for the infinite square-kagomé lattice we have extrapolated the data from table 1 to the thermodynamic limit according to the standard formulas for the two-dimensional spin-half HAFM (see, e. g. $[11,58,59])$, namely $e_{0}(N)=e_{0}(\infty)+A_{3} N^{-\frac{3}{2}}+\mathcal{O}\left(N^{-2}\right)$ for the ground state energy per bond, $m^{+}(N)=m^{+}(\infty)+B_{1} N^{-\frac{1}{2}}+\mathcal{O}\left(N^{-1}\right)$ for the order parameter, and $\Delta(N)=\Delta(\infty)+G_{2} N^{-1}+\mathcal{O}\left(N^{-\frac{3}{2}}\right)$ for the spin gap. In table 2 the results of 


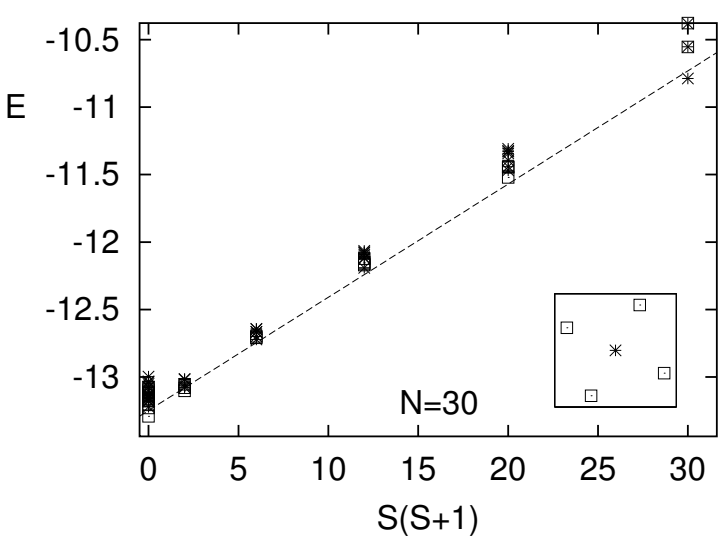

Figure 4. Low-energy spectrum for the HAFM on the square-kagomé lattice $(N=30)$ (the inset shows the $\mathbf{k}$ points in the Brillouin zone).

these extrapolations are presented and compared to those obtained for spin-half HAFM on the kagomé and on the star lattices. Our data suggest a small but finite spin gap and a vanishing order parameter.

The values of the extrapolated quantities of the square-kagomé lattice are very close to those of the kagomé lattice. Therefore, these data clearly yield evidence for a magnetically disordered quantum paramagnetic ground state of the spin-half HAFM on the square-kagomé lattice which is most likely similar to that of the kagomé lattice.

Table 2. Results of the finite-size extrapolation of the ground state energy per bond $e_{0}$, the order parameter $\mathrm{m}^{+}$and the spin gap $\Delta$ of the spin-half HAFM on the square-kagomé lattice. For comparison we also show results for the kagomé and star lattices taken from $[11,12]$. To see the effect of quantum fluctuations we present $m^{+}$scaled by its classical value $m_{\text {class }}^{+}$for the two ordered states shown in figure 2. (The negative, but very small, extrapolated values for the square-kagomé and the kagomé lattices are an artifact of the limited accuracy of the extrapolation. We interpret these negative values as vanishing order parameters.)

\begin{tabular}{lccc}
\hline lattice & square-kagomé & kagomé & star \\
\hline$e_{0}$ & -0.2209 & -0.2172 & -0.3091 \\
$\Delta$ & 0.052 & 0.040 & 0.380 \\
$m^{+} / m_{\text {class }}^{+}$ & -0.032 & -0.036 & 0.122 \\
\hline
\end{tabular}

\section{Magnetization process}

In this section we briefly discuss the magnetization versus field curve for some finite squarekagomé lattices. The magnetization $m$ is defined as $m=2\left\langle\hat{S}_{z}\right\rangle / N$. We focus on those finite lattices having optimal lattice symmetries, i. e. $N=24,30$. In the high field sector we are able to present also data for $N=48$ and $N=54$.

The results are shown in figure 5 . Due to the spin gap (see tables 1 and 2) one observes a small zero-field plateau. Clear evidence for a further plateau is found at $m=1 / 3$ which can be attributed to the presence of triangles [60]. Note that a $m=1 / 3$ plateau is also observed for the triangular $[11,60-64]$, the kagomé $[11,38,60,65-67]$ and the star $[11,12]$ lattices.

At the saturation field $h_{\mathrm{s}}=3$ a jump in the magnetization curve appears. The presence of this jump was discussed already in $[51,68]$ and is related to the existence of independent localized magnon states found for a class of strongly frustrated spin lattices [11,38,39,69,71] among them 


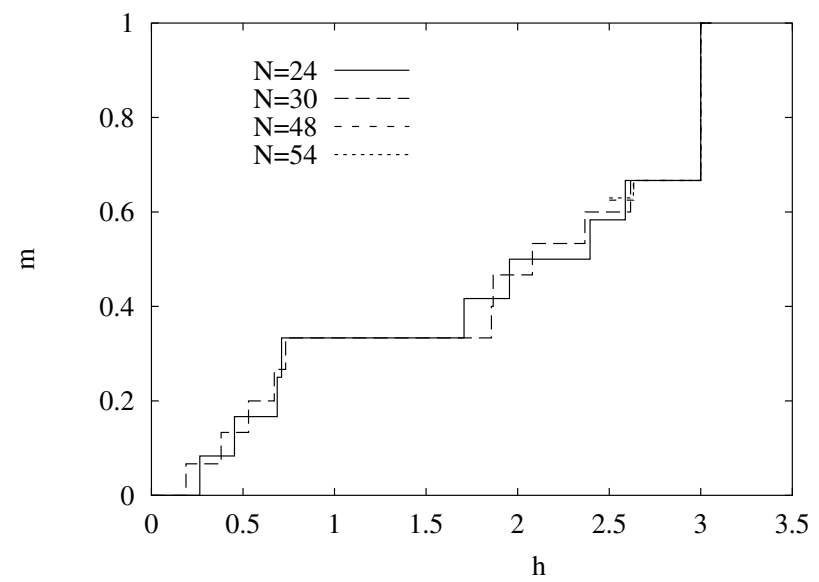

Figure 5. Magnetization curves of some finite spin-half HAFM systems on square-kagomé lattice in a magnetic field $(N=24,30,48,54)$.

the kagomé and the star lattices. In the case of the square-kagomé lattice these localized magnons live on the squares [71]. The height of the jump $\delta m$ is related to the maximum number $n_{\max }$ of independent localized magnons which can occupy the lattice. For the square-kagomé lattice we have $n_{\max }=N / 6$ and consequently $\delta m=1 / 3$. We mention that these localized magnon states are highly degenerate $[11,69-71]$. Just below the jump, i. e. at $m=2 / 3$ there is evidence for another plateau. Its width was estimated in [51] by finite size extrapolation to $\Delta h \approx 0.33 J$ for the infinite system.

\section{Low-temperature thermodynamics}

In this section we briefly summarize some specific features in the low-temperature thermodynamics which are related to the existence of the localized-magnon states. Let us first mention that in [50] an extra low-temperature maximum in the specific heat at zero magnetic field was discussed. Now we focus on the discussion of the high-field thermodynamics where the localized-magnon states become relevant. These localized-magnon states are not only responsible for the magnetization jump, see figure 5 in section 5 . They also may lead to a high-field spin-Peierls lattice instability [51], and they imply a residual ground-state entropy at the saturation field $h_{\mathrm{s}}$ [71]. Moreover, due to their huge degeneracy these states dominate the low-temperature thermodynamics in the vicinity of the saturation field [71].

From the experimental point of view the localized-magnon states manifest themselves most interestingly in a drastic change of the low-temperature specific heat, when the magnetic field passes the saturation field, and in the maximum of the isothermal entropy at saturation field leading to an enhanced magnetocaloric effect (for a general discussion of the magnetocaloric effect for quantum spin systems, see [70,72]). We illustrate this in figures 6 and 7 . In figure 6 we present the temperature dependence of the specific heat at three values of the external magnetic field, namely $h=0.95 h_{\mathrm{s}}, h_{\mathrm{s}}, 1.05 h_{\mathrm{s}}$. Obviously, the specific heat at the saturation field shows a conventional behavior with one broad maximum around $k T \approx J$. However, at fields slightly above/below the saturation field, the specific heat exhibits a well-pronounced extra low-temperature peak at about $k T \approx 0.05 J$.

In the context of magnetocalorics a residual entropy gives rise to large adiabatic cooling rates $\left(\frac{\partial T}{\partial h}\right)_{\mathrm{S}}=-\frac{T}{C}\left(\frac{\partial S}{\partial h}\right)_{\mathrm{T}}$ in the vicinity of the saturation field $[70,71]$. In figure 7 we consider the adiabatic cooling processes, i. e. we show curves of constant entropy as a function of magnetic field and temperature. It is obvious that lowering the field from $h>h_{\mathrm{s}}$ up to the saturation field $h_{\mathrm{s}}$ leads to an enhanced cooling of the magnet. 


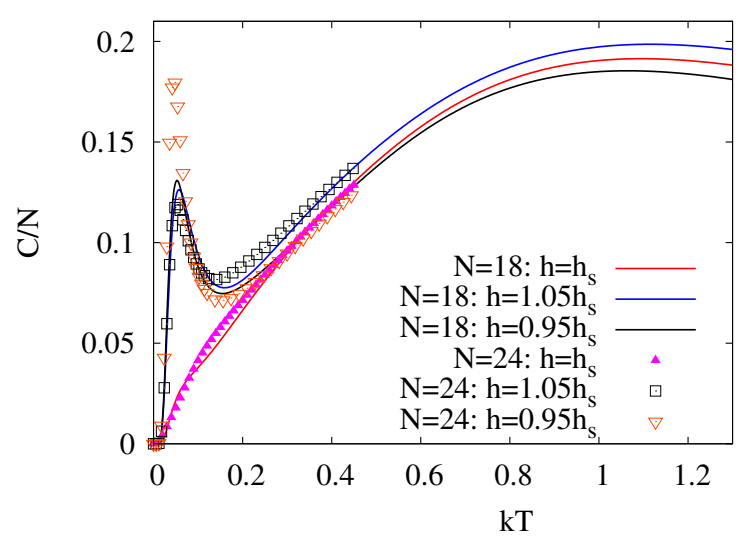

Figure 6. Specific heat per site $C / N$ versus temperature $T$ for $N=18$ (solid lines) and $N=24$ (symbols) sites and three values of the magnetic field $h=0.95 h_{\mathrm{s}}, h=h_{\mathrm{s}}, h=1.05 h_{\mathrm{s}}$. Note that for $N=24$ data only at low temperatures $T / h_{\mathrm{s}}<0.15$ are shown.

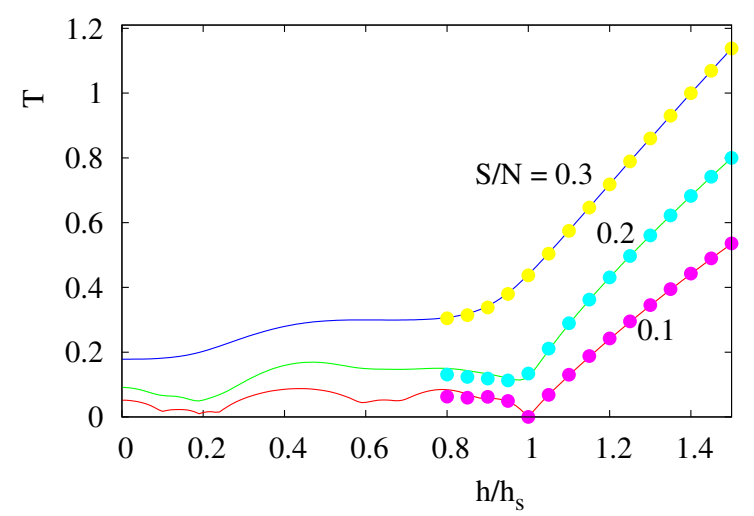

Figure 7. Constant entropy curves as a function of magnetic field $h$ and temperature $T$, $S(T, h, N) / k N=$ const, for the square-kagomé lattice with $N=18$ (solid lines) and $N=24$ (symbols) sites. Note that for $N=24$ data only at high fields $h / h_{\mathrm{s}}>0.8$ are shown.

\section{Summary and conclusions}

In this paper we have discussed the ground-state properties and the low-temperature thermodynamics near saturation field of the spin-half Heisenberg antiferromagnet on the square-kagomé lattice. This lattice has similarities with the kagomé as well as with the star lattice. The kagomé and the square-kagomé lattices have coordination number $z=4$ and are built by corner sharing triangles. The star lattice $(z=3)$ shares with the square-kagomé lattice the property of having two nonequivalent nearest-neighbor bonds and of having an even number (namely six) of sites per unit cell (note that the kagomé lattice has three sites per unit cell and all nearest-neighbor bonds are equivalent). On the classical and on the semiclassical level of linear spin wave theory the ground state of the Heisenberg antiferromagnet on all three lattices exhibits very similar properties. However, it was argued [12] that in the extreme quantum limit $s=1 / 2$ just these geometrical properties of the star lattice in common with the square-kagomé lattice but different to the kagomé lattice lead to different quantum ground states for the star and the kagomé lattices. Interestingly, our results for the square-kagomé lattice lead to the conclusion that the quantum ground state of the Heisenberg antiferromagnet on the square-kagomé lattice is similar to that of the kagomé lattice. 
We find evidence for a spin-liquid like ground state with a small gap of about $J / 20$ and a considerable number of low-lying singlets within this spin gap. Contrary to the star lattice case we do not see here a tendency towards forming a valence bond crystal ground state. The magnetization curve of the $s=1 / 2$ HAFM on the square-kagomé lattice shows a jump just below saturation and three plateaux at $m=0,1 / 3$ and $2 / 3$. The jump is related to the existence of localized eigenstates that built a highly degenerate ground-state manifold at the saturation field. The localized states give rise to an extra low-temperature maximum in the specific heat and a large magnetocaloric effect near the saturation field.

\section{Acknowledgement}

We thank A. Honecker for stimulating discussions. We acknowledge support from the Deutsche Forschungsgemeinschaft (Project Ri615/16-1) and from the Polish Committee for Scientific Research (Project No. 1 PO3B 108 27).

\section{References}

1. Frustrated Spin Systems, Diep H.T., ed. World Scientific, Singapore, 2004.

2. Quantum Magnetism, Schollwöck U., Richter J., Farnell D.J.J., Bishop R.F., Eds. Lecture Notes in Physics, 645. Springer, Berlin, 2004.

3. Taniguchi S., Nishikawa T., Yasui Y., Kobayashi Y., Sato M., Nishioka T., Kontani M., Sano K., J. Phys. Soc. Jpn., 1995, 64, 2758.

4. Kageyama H., Yoshimura K., Stern R., Mushnikov N.V., Onizuka K., Kato M., Kosuge K., Slichter C.P., Goto T., Ueda Y., Phys. Rev. Lett., 1999, 82, 3168.

5. Coldea R., Tennant D.A., Tylczyński Z., Phys. Rev. B, 68, 134424 (2003).

6. Tanaka H., Ono T., Katori H.A., Mitamura H., Ishikawa F., Goto T., Progr. Theor. Phys. Suppl., 2002, 145, 101.

7. Harrison A., J. Phys.: Condens. Matter, 2004, 16, S553.

8. Lemmnes P., Milet P. - In: Quantum Magnetism, Lecture Notes in Physics 645, Schollwöck U., Richter J., Farnell D.J.J., Bishop R.F., eds. Springer-Verlag, Berlin, 2004, p. 433-477.

9. Mendels P., Bert F., de Vries M.A., Olariu A., Harrison A., Duc F., Trombe J.C., Lord J.S., Amato A., Baines C., Phys. Rev. Lett., 2007, 98, 077204.

10. Helton J.S., Matan K., Shorres M.P., Nytko E.A., Bartlett B.M., Yoshida Y., Takano Y., Suslov A., Qiu Y., Chung J.-H., Nocera D.G., Lee Y.S., Phys. Rev. Lett., 2007, 98, 107204.

11. Richter J., Schulenburg J., Honecker A., - In: Quantum Magnetism, Lecture Notes in Physics 645, Schollwöck U., Richter J., Farnell D.J.J., Bishop R.F., eds. Springer-Verlag, Berlin, 2004, p. 85-183.

12. Richter J., Schulenburg J., Honecker A., Schmalfuß D., Phys. Rev. B, 2004, 70, 174454.

13. Schmalfuß D., Tomczak P., Schulenburg J., Richter J., Phys. Rev. B, 2002, 65, 224405.

14. Strečka J., Čanová L., Jaščur M., Hagiwara M., Phys. Rev. B, 2008, 78, 024427.

15. Yan-Zhen Zheng, Ming-Liang Tong, Wei Xue, Wei-Xiong Zhang, Xiao-Ming Chen, Fernande Grandjean, Gary J. Long, Angew. Chem. Int. Ed., 2007, 46, 6076.

16. Cave D., Coomer F.C., Molinos E., Klauss H.-H., Wood P.T., Angew. Chem. Int. Ed., 2006, 45, 803.

17. 21 Norman R.E., Stenkamp R.E., Acta Crystallogr., Sect. C: Cryst. Struct. Commun., 1990, 46, 6; Gonzalez M., Cervantes-Lee F., ter Haar L.W., Mol. Cryst. Liq. Cryst. Sci. Technol., Sect. A, 1993, 233, 317.

18. Chandra P., Doucot B., Phys. Rev. B, 1988, 38, R9335.

19. Schulz H.J., Ziman T.A.L., Europhys. Lett.,1992, 18, 355); Schulz H.J., Ziman T.A.L., Poilblanc D., J. Phys. I, 1996, 6, 675.

20. Richter J., Phys. Rev. B, 1993, 47, 5794.

21. Bishop R.F., Farnell D.J.J., Parkinson J.B., Phys. Rev. B, 1998, 58, 6394.

22. Sushkov O.P., Oitmaa J., Weihong Z., Phys. Rev. B, 2001, 63, 104420.

23. Capriotti L., Becca F., Parola A., Sorella S., Phys. Rev. Lett., 2001, 87, 97201.

24. Singh R.R.P., Zheng W., Oitmaa J., Sushkov O.P., Hamer C.J., Phys. Rev. Lett., 2003, 91, 017201.

25. Sirker J., Weihong Z., Sushkov O.P., Oitmaa J., Phys. Rev. B, 2006, 73, 184420. 
26. Darradi R., Derzhko O., Zinke R., Schulenburg J., Krüger S.E., Richter J., Phys. Rev. B, 2008, 78, 214415.

27. Isaev L., Ortiz G., Dukelsky J., Phys. Rev. B, 2009, 79, 024409.

28. Pardini T., Singh R.R.P., Phys. Rev. B, 2009, 79, 094413.

29. Schmidt R., Schulenburg J., Richter J., Betts D.D., Phys. Rev. B, 2002, 66, 224406.

30. Oitmaa J., Weihong Z., Phys. Rev. B, 2004, 69, 064416.

31. Schmalfuß D., Darradi R., Richter J., Schulenburg J., Ihle D., Phys. Rev. Lett., 2006, 97, 157201.

32. Viana J.R., de Sousa J.R., Continentino M.A., Phys. Rev. B, 2008, 77, 172412.

33. Yoshikawa T., Ogata M., Phys. Rev. B, 2009, 79, 144429.

34. Misguich G., Sindzingre P., J. Phys.: Condens. Matter, 2007, 19145202.

35. Lhuillier C., Sindzingre P., Fouet J.-B., Can. J. Phys., 2001, 79, 1525.

36. Moessner R., Can. J. Phys., 2001, 79, 1283.

37. Misguich G., Lhuillier C. - In: Frustrated Spin Systems, Diep H.T., ed. World Scientific, Singapore, 2004, p. 229-306.

38. Schulenburg J., Honecker A., Schnack J., Richter J., Schmidt H.-J., Phys. Rev. Lett., 2002, 88, 167207.

39. Richter J., Schulenburg J., Honecker A., Schnack J., Schmidt H.-J., J. Phys.: Condens. Matter, 2004, 16, S779.

40. Sachdev S. - In: Quantum Magnetism, Lecture Notes in Physics 645, Schollwöck U., Richter J., Farnell D.J.J., Bishop R.F., eds. Springer-Verlag, Berlin, 2004, p. 381-431.

41. Lecheminant P., Bernu B., Lhuillier C., Pierre L., Sindzingre P., Phys. Rev. B, 1997, 56, 2521.

42. Waldtmann Ch., Everts H.-U., Bernu B., Lhuillier C., Sindzingre P., Lecheminant P., Pierre L., Eur. Phys. J. B, 1998, 2, 501.

43. Mila F., Phys. Rev. Lett., 1998, 81, 2356.

44. Schmalfuß D., Richter J., Ihle D., Phys. Rev. B, 2004, 70, 184412.

45. Wang F., Vishwanath A., Phys. Rev. B, 2006, 74, 174423.

46. Singh R.R.P., Huse D., Phys. Rev. B, 2007, 76 180407(R).

47. Jiang H.C., Weng Z.Y., Sheng D.N., Phys. Rev. Lett., 2008, 101, 117203.

48. Hermele M., Ran Y., Lee P.A., Wen X.-G., Phys. Rev. B, 2008, 77, 224413.

49. Siddharthan R., Georges A., Phys. Rev. B, 2002, 65, 014417.

50. Tomczak P., Richter J., J. Phys. A: Math. Gen., 2003, 36, 5399.

51. Richter J., Derzhko O., Schulenburg J., Phys. Rev. Lett., 2004, 93, 107206.

52. Grünbaum B., Shephard G.C. Tilings and Patterns. W.H. Freeman and Company, New York, 1987.

53. Harris A.B., Kallin C., Berlinsky A.J., Phys. Rev. B, 1992, 45, 2899.

54. Chalker J.T., Holdsworth P.C.W., Shender E.F., Phys. Rev. Lett., 1992, 68, 855; Shender E.F., Holdsworth P.C.W. - In: Fluctuations and Order: a New Synthesis, M.M. Millonas ed. SpringerVerlag, 1996.

55. Asakawa H., Suzuki M., Physica A, 1994, 205, 687.

56. Leung P.W., Elser V., Phys. Rev. B, 1993, 47, 5459.

57. Bernu B., Lecheminant P., Lhuillier C., Pierre L., Phys. Rev. B, 1994, 50, 10048.

58. Neuberger H., Ziman T., Phys. Rev. B, 1989, 39, 2608.

59. Hasenfratz P., Niedermayer F., Z. Phys. B, 1993, 92, 91.

60. Honecker A., Schulenburg J., Richter J., J. Phys.: Condens. Matter, 2004, 16, S749.

61. Nishimori H., Miyashita S., J. Phys. Soc. Jpn., 1986, 55, 4448.

62. Honecker A., J. Phys.: Condens. Matter, 1999, 11, 4697.

63. Chubukov A.V., Golosov D.I., J. Phys.: Condens. Matter, 1991, 3, 69.

64. Farnell D.J.J., Zinke R., Schulenburg J., Richter J., J. Phys.: Condens. Matter, 2009, 21, 406002.

65. Hida K., J. Phys. Soc. Jpn., 2001, 70, 3673.

66. Cabra D.C., Grynberg M.D., Holdsworth P.C.W., Pujol P., Phys. Rev. B, 2002, 65, 094418.

67. Cabra C., Grynberg M.D., Holdsworth P.C.W., Honecker A., Pujol P., Richter J., Schmalfuß D., Schulenburg J., Phys. Rev. B, 2005, 71, 144420.

68. Schnack J., Schmidt H.-J., Richter J., Schulenburg J., Eur. Phys. J. B, 2001, 24, 475.

69. Zhitomirsky M.E., Tsunetsugu H., Phys. Rev. B, 2004, 70, 100403(R); Derzhko O., Richter J., Phys. Rev. B, 2004, 70, 104415; Zhitomirsky M.E., Tsunetsugu H., Prog. Theor. Phys. Suppl., 2005, 160, 361; Zhitomirsky M.E., Tsunetsugu H., Phys. Rev. B, 2007, 75, 224416.

70. Zhitomirsky M.E., Honecker A., J. Stat. Mech.: Theor. Exp., 2004, P07012.

71. Derzhko O., Richter J., Eur. Phys. J. B, 2006, 52, 23.

72. Schnack J., Schmidt R., Richter J., Phys. Rev. B, 2007, 76, 054413. 


\title{
Антиферомагнетик Гайзенберга на ґратці квадратне кагоме
}

\author{
Й. Ріхтер ${ }^{1}$, Й. Шуленбург ${ }^{2}$, П. Томчак ${ }^{3}$, Д. Шмальфус ${ }^{1}$ \\ ${ }^{1}$ Інститут теоретичної фізики, Університет Магдебурга, поштова скринька 4120, D-39016 Магдебург, \\ Німеччина \\ 2 Університетський обчислювальний центр, Університет Магдебурга, поштова скринька 4120, \\ D-39016 Магдебург, Німеччина \\ 3 Відділ фізики, Університет Адама Міцкевича, Умультовська 85, 61-614 Познань, Польща
}

Отримано 8 червня 2009 р.

Ми обговорюємо основний стан, низьколежачі збудження, а також термодинаміку у сильному полі антиферомагнетика Гайзенберга на двовимірній ґратці квадратне кагоме. Ця магнітна система належить до класу сильно фрустрованих спінових систем з безмежним нетривіальним виродженням класичного основного стану, так само, як і антиферомагнетик Гайзенберга на ґратці кагоме i на ґратці зірка. Квантовий основний стан спін-половина системи є квантовим парамагнетиком із скінченою спіновою щілиною та великим числом немагнітних збуджень всередині щілини. Ми також обговорюємо криву намагніченість-поле, у якій $є$ плато та макроскопічний стрибок намагніченості до значення насичення через стани, що називаються незалежними локалізованими магнонами. Ці локалізовані стани є сильно виродженими та зумовлюють цікаві риси низькотемпературної термодинаміки у сильному магнітному полі, такі як додатковий низькотемпературний пік у теплоємності і посилений магнітокалоричний ефект.

Ключові слова: фрустрація, квантовий антиферомагнетик Гайзенберга, квантовий безлад, сильне магнітне поле

PACS: $75.10 . J m, 75.45 .+j, 75.60 . E j, 75.50 . E e$ 
